\title{
Fourier Transform Infrared Spectroscopy Studies of Water-Polymer Interactions in Chemically Amplified Photoresists
}

\author{
Laurie A. McDonough ${ }^{\dagger}$, Viktor Chikan, Zee Hwan Kim, and Stephen R. Leone* \\ Departments of Chemistry and Physics, and Lawrence Berkeley National Laboratory, \\ University of California, Berkeley, California 94720 \\ William D. Hinsberg \\ IBM Almaden Research Center, 650 Harry Road, San Jose, California, 95120-6099 \\ *Corresponding author; email: $\underline{\text { srl@cchem.berkeley.edu }}$ \\ ${ }^{\dagger}$ Department of Chemistry and Biochemistry, University of Colorado, Boulder, Colorado \\ 80309-0440
}




\begin{abstract}
:
Fourier-Transform Infrared (FTIR) absorption spectroscopy is implemented to measure the infrared spectrum of water absorbed by the Poly(t-butoxycarbonylstyrene) (tBOC) and the ketal-protected Poly(hydroxystyrene) (KRS-XE) polymer photoresists. The shape and intensity of the $\mathrm{OH}$ stretching band of the water spectrum is monitored in a variety of humidity conditions in order to obtain information on the hydrogen-bonding interactions between the water and the polymer chains. The band is deconvoluted into four sub-bands, which represent four types of water molecules in different environments. Because of the hydrophilicity of the polymers studied, a large portion of the sorbed water molecules is believed to be strongly bound to the polar sites of the polymer. The ratios of each type of water are found to be dependent on the humidity conditions to which the sample was exposed. At higher humidities, there is an increase in the fraction of free and weakly-bound water molecules. These findings are used to explain the humidity dependence of the deprotection reaction rates, since certain types of water may slow transport of reactive species within the polymer network.
\end{abstract}




\section{INTRODUCTION}

Water sorption is an interesting process that can affect the physical, mechanical and chemical properties of a polymer. It is important to many polymer industries, including packaging, chemical sensors, drug delivery, artificial organs, and electronics. The rate and extent of the water uptake are determined by a variety of factors, such as temperature, humidity, polymer chemistry, film thickness, pore size and density, and material processing. ${ }^{1-4}$ The structure of the sorbed water is of particular importance, as it can give insight into the interactions between the water and the polymer substrate. Water is a unique penetrant because it interacts strongly with polar groups of the polymer and can form clusters that are confined by the polymer chains. Among the physical changes that water can induce are plasticization, hygrothermal degradation, swelling, alteration of tensile strength and hardness, and change in net dielectric constant of a film. ${ }^{2-6}$ Water can also affect chemical processes in polymers such as the deprotection reaction in photoresists used in the fabrication of electronic circuits. ${ }^{2,7,8}$

Poly(t-butoxycarbonylstyrene) (tBOC) ${ }^{9}$ and ketal-protected Poly(hydroxystyrene) $(\mathrm{KRS}-\mathrm{XE})^{10}$ are two important chemically amplified photoresist systems. The tBOC polymer, shown in Figure 1a, is a thin film cast with a photoacid generator (PAG). Exposure to UV radiation forms the acid, which reacts with the tBOC in the presence of heat and is converted to polyhydroxystyrene (PHOST). The tBOC film is initially relatively dry, but as the polymer is modified it becomes more hydrophilic and rapidly absorbs water. The amount of water absorbed by the photoresist depends on the local 
humidity. Burns et al. ${ }^{7}$ have found that an increase in the humidity will slow the deprotection reaction. This result was unexpected. They hypothesize a "pH leveling" effect in which water reacts with photogenerated acid, decreasing the strength of the acid and lowering the reaction rate. This work provides an additional, complementary explanation.

The chemistry for the KRS-XE system is different. ${ }^{7,10}$ The initial exposure occurs in vacuum, while the post exposure processing takes place in a clean room where the humidity is carefully controlled. As shown in Figure 1b, a stoichiometric amount of water is required for the solubility switching reaction. The polymer is initially hydrophilic and absorbs the water needed for the deprotection. The activation energy is low, so a post-exposure bake is unnecessary. Burns et al. found that increased humidity

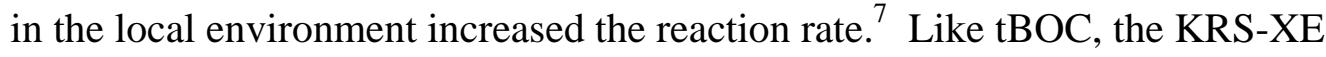
deprotection reaction results in the polymer PHOST. However, the resulting films are not identical, because the composition and quality of the film depends on the processing methods used. Also, the percentage of the polymer that is converted may be different in each case.

Fourier-Transform Infrared (FTIR) spectroscopy has produced insights into the structure of water in polymer films. ${ }^{3,5,11-16}$ The vibrational spectrum can be measured in situ at a variety of humidities and provides details about the water-polymer interactions and hydrogen bonding. In this investigation, the molecular information obtained contributes to the understanding of transport through the polymer, which ultimately helps explain the deprotection reaction kinetics. 


\section{EXPERIMENT}

\section{A. Materials}

Polymer solutions were prepared using a previously described method ${ }^{7}$ and spun onto $25 \mathrm{~mm}$ sapphire wafers. The resulting tBOC photoresist film is $990 \mathrm{~nm}$ thick. The modified tBOC, or PHOST sample, was prepared by exposing a tBOC film to a 100 $\mathrm{mJ} / \mathrm{cm}^{2}$ dose of $257 \mathrm{~nm}$ ultraviolet light. The wafer was then baked at $100{ }^{\circ} \mathrm{C}$ for 90 seconds. The thickness of the resulting film is approximately $635 \mathrm{~nm}$.

The KRS-XE photoresist was also spun onto $25 \mathrm{~mm}$ sapphire wafers, but to a thickness of $370 \mathrm{~nm}$. To prepare the modified polymer sample, the resist was exposed to a dose of $100 \mathrm{~mJ} / \mathrm{cm}^{2}$ of ultraviolet light. After 5 minutes of exposure to clean room conditions with controlled humidity, the thickness was $335 \mathrm{~nm}$.

\section{B. Instrument}

Figure 2 shows the apparatus used for the experiments. It consists of a modified Bruker Optics IFS 66v/s Fourier Transform Infrared (FTIR) spectrometer with a tungsten IR source and a liquid nitrogen cooled MCT (HgCdTe) detector. Both the sample and instrument chambers can be pumped down to $2.0 \mathrm{kPa}$. The sample chamber is isolated by vacuum flanges with sapphire windows to allow the passing of the infrared radiation. Gas inlets and outlets were added to the faceplate of the sample chamber to allow the sample environment to be humidity controlled. Dry nitrogen is used to purge the sample. The nitrogen can be passed through a reservoir containing water to produce wet nitrogen. Mixing different ratios of wet nitrogen and dry nitrogen produces the variety of humidities used in this work, which are recorded by a thermohygrometer at the gas outlet. 
Samples are loaded onto a holder with mounting positions for two samples. An external manipulator can move samples into the IR beam, as well as move them out of the way to allow for a background scan. For the experiments performed here, the two samples mounted at the same time are the modified and unmodified polymers for either the tBOC or the KRS-XE system. The background scan is taken immediately before or after the sample scan in order to best match the humidity conditions and allow for elimination of the gas phase water features in the spectrum. The final spectra, which show only the absorbance of the water sorbed into the polymer, are obtained through the following subtraction:

$$
A=\log \left(\frac{I_{0}}{I}\right)-\log \left(\frac{I_{0}^{\prime}}{I^{\prime}}\right),
$$

where $I_{0}$ is the background spectrum under humid conditions, $I$ is the spectrum of the

sample in humid conditions, $I_{0}^{\prime}$ is the background spectrum in dry conditions, and $I^{\prime}$ is the spectrum of the sample in dry conditions.

\section{RESULTS AND DISCUSSION}

\section{A. tBOC system}

Spectra of water sorbed in the modified tBOC polymer were obtained at $10 \%, 21 \%$, $37 \%, 67 \%$ and $79 \%$, all $\pm 2 \%$, relative humidity (RH). Figure 3a shows the $\mathrm{OH}$ stretching region of these spectra, which is the most sensitive region to hydrogen bonding (H-bonding), with the arrow pointing in the direction of increasing humidity. The band can be deconvoluted into distinct sub-bands within the $\mathrm{OH}$ stretching band, which reflect different types of water molecular environments. ${ }^{3,11,14,15,17}$ This type of analysis was 
performed on our sorbed water spectra. We chose four sub-bands and designated them strongly-bound, medium-bound, weakly-bound and free water. Previous literature indicates that the water is molecularly absorbed rather than dissociated. ${ }^{14,15,18}$ The results for modified tBOC are shown in Figure 3b.

The lowest frequency band is commonly attributed to the most strongly bound water molecules. ${ }^{3,5,11,14-17,19}$ This peak at $2953 \mathrm{~cm}^{-1}$ is due to water molecules H-bonded onto specific polar sites along the polymer network. In the tBOC photoresist system, the $\mathrm{OH}$ group formed during the deprotection would be a good candidate for a strong H-bonding site. The strongly-bound peak is usually the broadest, due to heterogeneity of the bonding sites. The largest sub-band, found at $3257 \mathrm{~cm}^{-1}$ corresponds to medium-strength hydrogen bonds. These water molecules are still associated with the polymer chains, but they are not as tightly hydrogen-bonded as the strongly bound ones. In the case of modified tBOC, we believe the medium and strongly-bound bands are larger because of the hydrophilicity of the polymer. The band at $3513 \mathrm{~cm}^{-1}$ shows weakly-bonded water molecules, and the highest frequency peak at $3623 \mathrm{~cm}^{-1}$ is characteristic of free or unassociated water. These less-bound water molecules can be found in the pores formed by spaces between the polymer chains. The bands are narrower, and because the water molecules are relatively isolated, there is less heterogeneous broadening.

The fraction of each type of water is calculated by integrating the indicated sub-band and dividing by the area of the total $\mathrm{OH}$ stretching band from $2500-3750 \mathrm{~cm}^{-1}$. However, it is not very straightforward to convert these fractions into actual amounts of water molecules. The molar absorptivity significantly increases with the degree of hydrogen bonding, so the cross section would be different for each sub-band. .,11,14 $^{-1}$ 
Therefore, we look only at the change in each fraction with humidity to see how the interaction inside the polymer network is affected when more water is present. The fraction of each type of water present depends on various chemical and physical properties of the polymer, such as structure, cross-linking, molecular weight, pore size and density.,11,13,16,20

The intensity of all four sub-bands, and therefore the amount of each type of water, increases with local humidity. However, the fraction of each of the four sub-bands changes with humidity in a different way, as illustrated in Figure 4. Error bars represent one standard deviation from multiple measurements. The deviations are due to the reproducibility of the experiment, particularly because of drift in the humidity conditions. The accuracy of the spectral fit also plays a role.

The relative amounts of free and weakly bound water molecules increase more rapidly than the medium and strongly-bound water. This is manifested by an increase in the fraction of these subbands with increasing humidity, as seen in Figure 4. For example, the weakly-bound water changes from a fraction of $0.04 \pm 0.01$ at $10 \% \mathrm{RH}$ to $0.08 \pm 0.01$ at $79 \% \mathrm{RH}$. The fraction of medium-bound water remains fairly constant with humidity, and the fraction of strongly-bound water decreases.

Similar behavior has been observed in other polymers and can be explained by the following considerations. ${ }^{2,5,6,11,14,15,18}$ Enthalpy mandates that at the lower humidities, when there is less water available, the absorbed water molecules would be more strongly bound. Water hydrogen bonds to polar sites along the polymer chains. As the sites for water association begin to saturate, the water molecules increasingly tend towards locations in the pores formed by spaces between polymer chains. They also begin to 
come together to form dimers and larger clusters. Although there is no direct spectral evidence for dimer and cluster formation, indirect evidence exists. As described above, the increase in fraction of free and weakly-bound water signals the saturation of sites for direct hydrogen bonding to the polymer. At this point water molecules are most likely to start hydrogen bonding to each other. ${ }^{2}$ It is important to note that the absolute amount of each water species increases with relative humidity. Therefore, even though changes in fraction may appear small in Figure 4, they can indicate a significant shift in the overall environment.

When water forms clusters in polymer chains, the rates of transport of water and other species in the film can be decreased. ${ }^{6,14,18,21}$ Berger et al. ${ }^{2}$ found that in the case of PHOST, diffusion is impeded when there is one water molecule present for every two $\mathrm{OH}$ sites in the polymer. This is when the hydrogen bonding to hydrophilic sites is saturated enough that water molecules begin to cluster together. The clustering of water molecules may serve to slow the diffusion of the photoacid species. Interaction with water could decrease the mobility of the large acid counter ion and limit transport to active sites. ${ }^{22}$ Therefore, in addition to the dilution of the acid by the water ${ }^{7}$, this limiting of transport within the polymer may be an additional cause for the slowed reaction rate with increasing humidity reported by Burns, et al.

Similar experiments were carried out on unmodified tBOC films. The tBOC is hydrophobic and has been reported to take up less than one fourth as much water as PHOST, the modified polymer. ${ }^{7}$ In our experiments, it was difficult to measure the spectra because of the small amounts of water present and the low signal-to-noise ratio caused by error in the subtraction of the gas phase water signal. ${ }^{23}$ Therefore, we were not 
able to carry out an analysis of the different water environments on this polymer. We believe that the water molecules would be less strongly-bound than the waters in the modified polymer because of the lack of hydrophilic sites on the polymer chain of unmodified tBOC.

\section{B. KRS-XE}

Spectra of the water in the KRS-XE system, measured at $12 \%, 21 \%, 41 \%$, and $51 \%$ (all $\pm 2 \%$ ) relative humidity, are shown in Figure 5 a. In this system, the original polymer needs to absorb water so that it is available as reactant in the deprotection reaction. After the reaction is carried out, the polymer is slightly more hydrophilic and holds more water. This is illustrated in Figure 5a. The adjacent solid and dotted lines are spectra of the unmodified and modified polymers at the same humidity, and the arrow points in the direction of increasing humidity. The absorbance is higher for the modified polymer, denoting a greater amount of water present. The shape of the band is similar to the shape of the water band in the modified tBOC, as expected because of the similarity of the chemical structures of these polymers.

Figure 5b was constructed in a similar fashion to Figure 4 and illustrates the changing shape of the water band with humidity. The KRS-XE system follows a similar pattern to the tBOC system, in that the fractions of free and weakly bound water seem to increase as the medium and strongly-bound water molecule fractions decrease. For the unmodified polymer, there is a big change from $12 \% \mathrm{RH}$ to $21 \% \mathrm{RH}$, and then the fractions stay fairly constant with humidity. This is an interesting point because Burns et al. found that the deprotection reaction rate increases with humidity until the humidity reaches $25 \%$, and 
then it stays constant. ${ }^{7}$ Because water is a reactant in the deprotection, the leveling off of the reaction rate can occur when there is an excess of water, meaning the polymer becomes the limiting reagent. Transport could also play a role. As with the modified tBOC, at higher humidities the free and weakly-bound water molecule fractions are increasing because the available hydrophilic sites are becoming saturated. Therefore, it is possible that water molecules are clustering, which may slow diffusion of water and/or the acid to available reactive sites.

In the modified KRS-XE polymer, the increase in free and weakly-bound water species and the decrease in strongly-bound species may continue with increasing humidity beyond 21\%RH. Another interesting aspect of Figure $5 \mathrm{~b}$ is that in the unmodified polymer, the fraction of free water is greater than the fraction of weaklybound water. The opposite is true of the modified polymer. This is evidence that a subtle change in the polymer structure can change the water-polymer interactions.

\section{CONCLUSIONS}

This work has provided information regarding the water - polymer interactions in hydrated chemically amplified photoresists. The sorption of water in the polymer films is found to be highly dependent on the humidity of the sample environment. The $\mathrm{OH}$ stretching band, found at $2500-3750 \mathrm{~cm},{ }^{-1}$ deconvolutes into four sub-bands, which represent four different types of water present in the wet photoresists. These water molecules undergo varying degrees of hydrogen bonding to the polymer chains. The amount of each type of water is humidity dependent, with the fraction of free and weaklybound water increasing, and the fraction of medium and strongly-bound water 
decreasing, with increasing humidity. In the case of the tBOC photoresist system, this increase in free and weakly bound water probably causes slowed transport of acid to reaction sites on the polymer, resulting in a decreased deprotection reaction rate. In the case of the unmodified KRS-XE photoresist, similar changes occur at low humidites, but the fractions stay relatively constant with increasing humidity after $21 \% \mathrm{RH}$. This mirrors the trend of the deprotection reaction rate with humidity, and it provides additional evidence that the polymer-water interactions may affect transport and ultimately reaction dynamics within a polymer network.

\section{ACKNOWLEDGEMENTS}

We gratefully acknowledge the support of this research by the National Science Foundation, through the Division of Materials Research. Construction of the laboratories and additional equipment support were provided by the Director, Office of Science, Office of Basic Energy Sciences, Chemical Sciences, Geosciences and Biosciences Division, U.S. Department of Energy under Contract No. DE-AC03-76SF00098. We would also like to thank the National Institute of Standards and Technology for a loan of some equipment used in this study. 


\section{REFERENCES}

1 R. Buchold, A. Nakladal, G. Gerlach, M. Herold, G. Gauglitz, K. Sahre, and K. Eichhorn, Thin Solid Films 350, 178 (1999); P. Neogi, Diffusion in Polymers. (Marcel Dekker, Inc, New York, 1996); A. Arce, F. Fornasiero, O. Rodriquez, C.

J. Radke, and J. M. Prausnitz, Phys. Chem. Chem. Phys. 6, 103 (2004).

2

C. M. Berger and C. L. Henderson, Polymer 44, 2101 (2003).

3

C. Mura, J. Yarwood, R. Swart, and D. Hodge, Polymer 42, 4141 (2001).

4

K. A. Schult and D. R. Paul, Journal of Applied Polymer Science 61, 1865 (1996).

5 S. Cotungo, D. Larobina, G. Mensitieri, P. Musto, and G. Ragosta, Polymer 42, $6431(2001)$.

$6 \quad$ P. Sutandar, D. J. Ahn, and E. I. Franses, Macromolecules 27, 7316 (1994).

7 S. D. Burns, D. R. Medeiros, H. F. Johnson, G. M. Wallraff, W. D. Hinsberg, and C. G. Willson, Proc SPIE 4690, 321 (2002).

8 O. Suga, H. Yamaguchi, and S. Okazaki, microelectronic engineering 13, 65 (1991); Y. Shibayama and M. Saito, Jap. J. Appl. Phys. 29 (10), 2152 (1990).

9 G. Wallraff, J. Hutchinson, W. Hinsberg, F. Houle, P. Seidel, R. Johnson, and W. Oldham, J. Vac. Sci. Technol. B 12 (6), 3857 (1994). D. R. Medeiros, A. Aviram, C. R. Guarnieri, W.-S. Huang, R. Kwong, C. K. Magg, A. P. Mahorowala, W. M. Moreau, K. E. Petrillo, and M. Angelopoulos, IBM J. Res. Dev. 45 (5), 639 (2001). 
C. Sammon, C. Mura, J. Yarwood, N. Everall, R. Swart, and D. Hodge, J. Phys. Chem. B 102, 3402 (1998).

Y. Maréchal and A. Chamel, J. Phys. Chem. 100, 8551 (1996).

Q. T. Nguyen, E. Favre, Z. H. Ping, and J. Néel, J. Membrane Sci. 113, 137 (1996).

H. Kitano, K. Ichikawa, M. Fukuda, A. Mochizuki, and M. Tanaka, J. Colloid Interface Sci. 242, 133 (2001).

Z. H. Ping, Q. T. Nguyen, S. M. Chen, J. Q. Zhou, and Y. D. Ding, Polymer 42, 8461 (2001).

M. Kawagoe, M. Takeshima, M. Nomiya, J. Qiu, M. Morita, W. Mizuno, and H. Kitano, Polymer 40, 1373 (1999).

Y. Maeda and H. Kitano, Spectrochimica Acta A 51, 2433 (1995).

C. Sammon, C. Deng, C. Mura, and J. Yarwood, J. Mol. Liquids 101 (1-3), 35 (2002).

K. Ichikawa, T. Mori, H. Kitano, M. Kukuda, A. Mochizuki, and M. Tanaka, J. Polym. Sci. Part B: Polym Phys 39, 2175 (2001).

Y. Maeda, M. Ide, and H. Kitano, J. Mol. Liquids 80, 149 (1999).

H. W. Starkweather, Macromolecules 8 (4), 476 (1975).

G. M. Wallraff and W. D. Hinsberg, Chem. Rev. 99, 1801 (1999).

Much of the noise comes from the subtraction of the gas phase water. If the local humidity conditions present during the measurement of the $\mathrm{I}$ and $\mathrm{I}_{0}$ spectra are not exactly the same, subtraction noise due to gaseous species is introduced. 
Performing more averages only gives a greater chance that the local humidity that the sample is exposed to will drift and cause more noise. 


\section{Figure Captions:}

Figure 1: Deprotection reactions of polymer systems studied: a) Poly(tbutoyxylcarbonyloxystyrene) (tBOC) b) Ketal-protected Poly(hydroxystyrene) (KRS$\mathrm{XE})$

Figure 2: Schematic diagram of FTIR spectrometer apparatus.

Figure 3: Spectra of water absorbed by the modified tBOC system. a) Black lines show spectra taken in modified polymer at 10, 21, 37, 67 and $79 \% \mathrm{RH}$. The arrow points in the direction of increasing humidity. b) Deconvoluted spectrum of $\mathrm{OH}$ stretching band at 67\% RH. Four sub-bands, which represent four different water environments, are shown by dotted lines. The sub-band with a peak at $2953 \mathrm{~cm}^{-1}$ represents strongly-bound water molecules, $3257 \mathrm{~cm}^{-1}$ is medium-bound, $3513 \mathrm{~cm}^{-1}$ is weakly-bound, and $3623 \mathrm{~cm}^{-1}$ shows free water molecules.

Figure 4: Fraction of total $\mathrm{OH}$ stretching band vs. relative humidity for the four subbands representing strongly-bound ( $\boldsymbol{\nabla})$, medium-bound $(\boldsymbol{\bullet})$, weakly-bound $(\boldsymbol{\Delta})$ and free $(\bullet)$ water molecules in the modified tBOC polymer sample of Figure 3.

Figure 5: Results of KRS-XE experiments. a) Spectra of absorbed water taken at $12 \%$, $21 \%, 41 \%$ and $51 \%$ relative humidity. Solid lines show spectra of the unmodified polymer; dotted lines show the modified polymer. The arrow points in the direction of 
increasing humidity. b) Fraction of total $\mathrm{OH}$ stretching band vs. relative humidity for the four sub-bands representing strongly-bound (₫), medium-bound ( $\bullet$ ), weakly-bound ( $\Delta$ )and free $(\bullet$ ) water molecules for the unmodified polymer. c) Fraction of total $\mathrm{OH}$ stretching band vs. relative humidity for the modified polymer. 


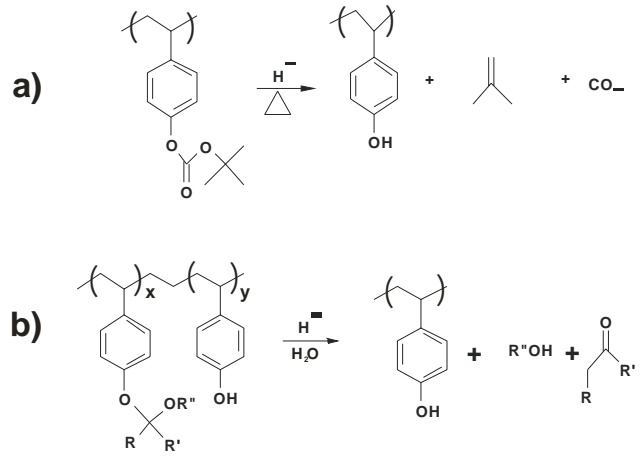

Figure 1 


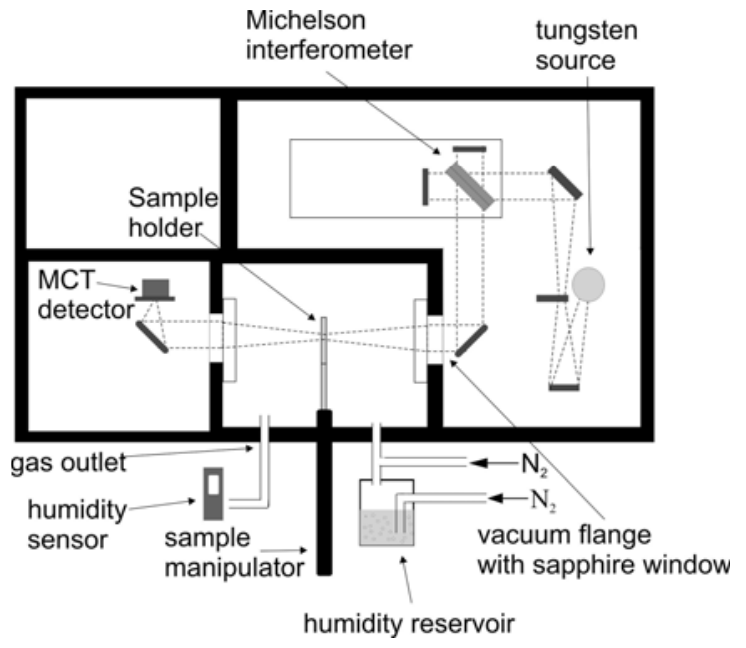

Figure 2 

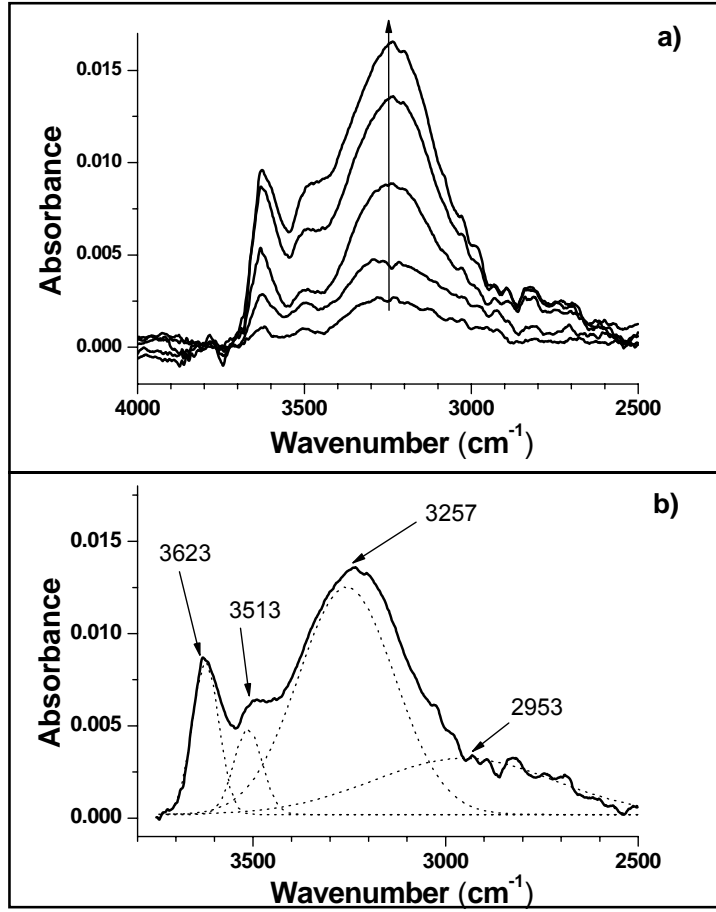

Figure 3 


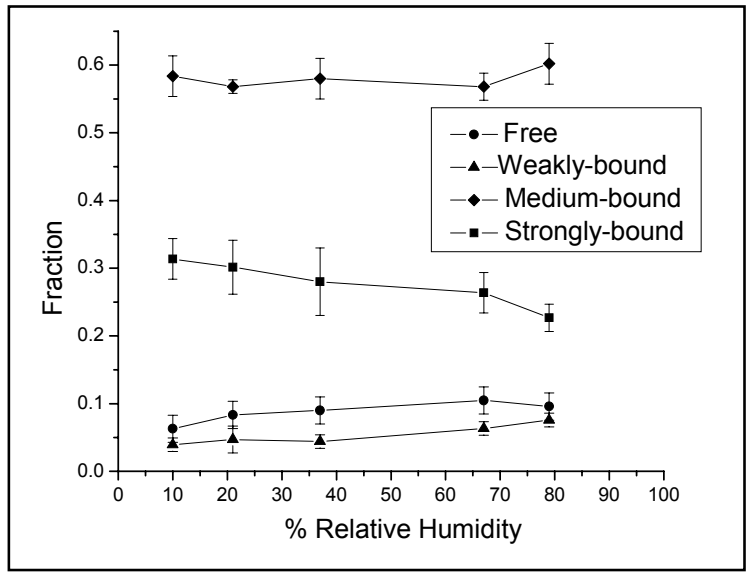

Figure 4 


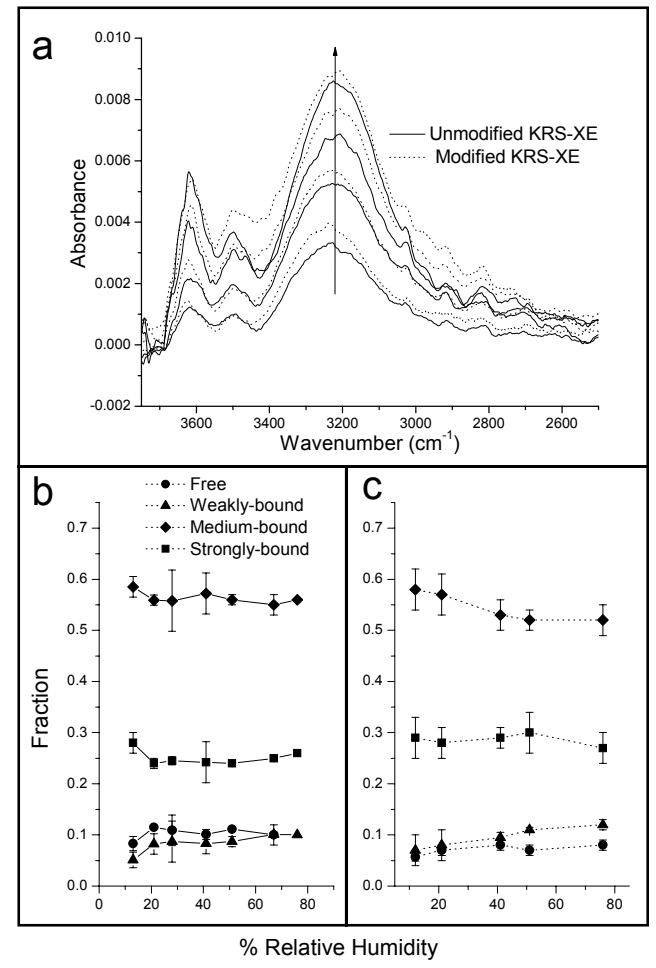

Figure 5 